Volumen: 22 Fecha de recibido: 17/10/2019 Fecha de publicación: Enero-Junio de 2020

Año: 2020 Fecha de aceptado: 27/11/2019 Correo: revista.societas@up.ac.pa

Numero: 1 Número de Páginas: 54-67 URL:https://revistas.up.ac.pa/index.php/societas

\title{
IMPORTANCIA DE LA CULTURA ORGANIZACIONAL EN LAS INSTITUCIONES EDUCATIVAS
}

IMPORTANCE OF ORGANIZATIONAL CULTURE IN EDUCATIONAL INSTITUTIONS

\section{Edith Yaqueline Burbano Pérez.}

Universidad Metropolitana de Educación Ciencia y Tecnología. Facultad de Educación. Doctorado en Ciencias de la Educación. Panamá, Panamá. Correo: jackytambo@gmail.com

\section{RESUMEN}

Es de gran relevancia la puesta en marcha de una Cultura Organizacional en el contexto educativo, porque permite desarrollar una serie de valores institucionales, que llevan a sus miembros a identificarse con una política o filosofía para promover un trabajo interdisciplinario, orientado a la consecución de unas metas corporativas. El objetivo de este artículo es exponer la importancia de la cultura organizacional en el desarrollo de las diferentes instituciones educativas, precisadas en el reconocimiento de los trabajos investigativos y documentos definidos para este estudio.

En la parte metodológica, se hizo revisión organizada de una serie de trabajos de investigación y textos de estudio, consultando la base de datos de Google académico, Dialnet y la biblioteca de la Universidad UMECIT, para lo cual, se procedió a la revisión bibliográfica de los documentos seleccionados sobre la temática tratada.

Se seleccionaron 10 trabajos de investigación: 3 tesis, 6 artículos científicos y 1 libro, obtenidos de las fuentes mencionadas anteriormente. Los documentos revisados determinan que la cultura organizacional es relevante en el desarrollo de las instituciones educativas, quienes deben tener presente el citado tema, debido a que es fundamental para el desarrollo de las diferentes 
instituciones las cuales deben promover valores sociales, éticos, morales y la filosofía institucional propia para la construcción de una sociedad más humana.

Palabras clave: Cultura Organizacional, Globalización, Proceso de Enseñanza- Aprendizaje, Escuela, Desarrollo Institucional.

\begin{abstract}
The implementation of an Organizational Culture in the educational context is of great relevance, because it allows the development of a series of institutional values, which lead its members to identify with a policy or philosophy to promote interdisciplinary work, oriented to the achievement of corporate goals. The objective of this article is to expose the importance of organizational culture in the development of different educational institutions, specified in the recognition of research work and documents required for this study.
\end{abstract}

In the methodological part, an organized review of a series of research papers and study texts was done, consulting the academic Google database, Dialnet and Umecit's library, for which, the bibliographic inspection of the selected documents was done of the topic to work.

Ten research papers were selected: 3 theses, 6 scientific articles and 1 book, obtained from the mentioned sources. The documents reviewed determine that the organizational culture is relevant in the development of educational institutions, which must keep in mind the mentioned topic, because it is fundamental for the development of the different institutions which must promote social, ethical and moral values and the institutional philosophy for the construction of a more humane society.

Keywords: Organizational Culture, Globalization, Teaching-Learning Process, School, Institutional Development. 


\section{INTRODUCCIÓN}

Con el pasar del tiempo, el ser humano como ser elemental de la sociedad, encuentra la necesidad de desarrollar maneras afables de ejecutar las actividades a través de alguna forma de organización. De igual manera las instituciones u organizaciones para ser consideradas como tal, se ven supeditadas a ordenar las actividades de sus integrantes, llegando a condicionar imagen y funcionamiento con originalidad y autonomía, en la búsqueda de satisfacer en forma adecuada y eficaz necesidades reciprocas entre clientes internos y externos, ante esto, se destaca como una condición para el caso, la organización laboral de las personas con el objetivo de mejorar el desempeño y lograr la satisfacción en la organización al mismo tiempo que abarca las necesidades del ser humano (Barney, 1986).

En el contexto educativo se reconoce la necesidad de fortalecer valores morales y éticos, así como métodos que se utilizarán en la organización, los que deberán estar en relación con la filosofía institucional para promover de tal manera el accionar de cada uno de los miembros en la institución, puesto que, el servicio que se ofrece a la comunidad es la educación, un bien que requiere de planificación constante, sin dejar de lado la evaluación institucional, la retroalimentación y la innovación (Sánchez \& De la Garza, 2013).

Actualmente se concede gran importancia al factor humano en las diferentes instituciones que tienen como finalidad la creación de bienes y servicios. Lo citado motiva a desarrollar el estudio de la cultura, por ser un elemento que otorga respuestas a muchos interrogantes presentados a partir de una serie de acontecimientos en el contexto de una organización (De Medina, 2008). La cultura simboliza y manifiesta todo aquello que es creado y aprendido por el ser humano (Schein, 2004). Por lo tanto, todas las personas y especialmente las comunidades, tienen culturas diferentes a las demás.

Smircich (1983) establece que los estudios sobre la cultura organizacional en el contexto general de las instituciones, constituye una contribución al desarrollo de las Ciencias Sociales, a pesar 
de que existen teorías y estudios en menor proporción sobre cultura organizacional aplicada al contexto educativo (Fernández, 2002). Finalmente, algunas de las teorías de la cultura organizacional plantean sus estudios con base en la antropología. Toca y Carillo (2009) expusieron la importancia de enfatizar en la cultura, para evaluar la eficiencia de una organización, debido a que esta requiere proyección y direccionamiento de las relaciones humanas para su desarrollo en contexto.

Con base en lo anotado, desde el punto de vista teórico y práctico, la cultura organizacional es necesaria porque permite adentrarse en el conocimiento de una institución para adecuarla y encaminarla al mejoramiento, siempre en beneficio y servicio de los demás, de ahí que la posición del docente en aceptar la aplicación de la cultura organizacional como estrategia para el mejoramiento de una organización educativa, es categórica, en la consecución de grandes metas u objetivos en beneficio institucional y de las personas que trabajen allí.

La cultura organizacional y su importancia en las instituciones educativas es concluyente para la consecución de la calidad en educación y el aumento de la misma, de ahí su relevancia en ser aplicada, además de promovida dentro de las diferentes instituciones educativas, puesto que de ella depende en cierta medida el desarrollo de la misión y visión de dichos organismos, quienes en última instancia necesitan desarrollar tal cultura para el perfecto cumplimiento de objetivos institucionales, ejemplo de ello, son los colegios religiosos, algunas universidades públicas o privadas, quienes desarrollan políticas internas con valores institucionales a través de una bien planeada cultura organizacional en beneficio de la comunidad académica en general.

El propósito de esta revisión bibliográfica es exponer la importancia de la cultura organizacional en el desarrollo de las diferentes instituciones educativas, precisadas en el reconocimiento de los trabajos investigativos analizados para este artículo.

\section{FUNDAMENTACIÓN TEÓRICA}


En el desarrollo de las instituciones, dicha cultura reviste gran importancia en el propósito de lograr los objetivos establecidos por éstas. Autores como Arévalo, Navarro y Bayona (2016) plantean que el Diccionario de Ultimate Business de Cambridge define la cultura organizacional como: "los tipos de actitudes y formas de trabajo acordadas entre los empleados de una empresa u organización" (Cambridge University Press, 2017). Además, Schein (2009 citado en Arévalo et al, 2016) define el anterior término como el modelo de hipótesis implícitas, distribuidas y aprendidas por un grupo de personas, luego de resolver los problemas de adaptación externa y de integración interna, el cual se ha desarrollado lo suficientemente bien para ser validado y enseñado en forma correcta a los integrantes de una comunidad, con la finalidad de que sean percibidos, pensados y aceptados en relación con tales variables.

Aunado a lo anterior, Peters y Waterman (1984 citados en Arévalo et al, 2016) sostienen que la cultura organizacional es aquella reunión dominante y relacionada de valores que se comparten y se transmiten por medio de significados simbólicos como: cuentos, mitos, leyendas, slogans y anécdotas, etc., es decir, hace referencia a todo lo simbólico que adquiere importancia debido al significado socio-cultural otorgado por una comunidad en particular.

Por su parte, Hofstede (citado por Ruíz y Naranjo, 2012) define el mencionado concepto como "programa mental colectivo que distingue a los miembros de un grupo o categoría de otro. Es compartida por personas cuya vivencia está dentro del mismo ambiente social donde fue aprendida" (p. 15). Ante ello, en forma sintética, la cultura organizacional es un programa instaurado en el constructo mental de las personas pertenecientes a una comunidad, agrupación, colectividad, institución educativa, universidad o empresa, que permite a cada uno de sus miembros identificarse a través del desarrollo de una filosofía institucional aceptada y promovida por todas los partes implicadas, una vez aplicada se constituirá en la columna vertebral de la organización y su puesta en práctica redundará en beneficio de la misma. 
Acontece que, Rodríguez (2009) expone que la cultura organizacional expresa los rasgos característicos aprendidos durante el desarrollo de una experiencia aprendida en un grupo, cuyas cualidades son aprendidas por todas las personas. A juicio de Schein (citado en Rodríguez, 2009) la cultura organizacional se encuentra en un nivel "más profundo" (p. 8) de las suposiciones más elementales o básicas que sean compartidas. Ante ello, la cultura organizacional dentro de las instituciones educativas reviste total importancia debido que el urdimbre de significaciones que son producto del acto socio- cultural deben ser conocidos y compartidos por todos los miembros de la comunidad u organización educativa, por eso, la relevancia de promocionar la cultura que refleja la idiosincrasia de la comunidad a la cual se pertenece.

Además de ello, Schein (1988) estima relevante que la cultura organizacional contribuya a la incorporación interna de las personas por medio de la relación de cada uno de sus miembros e incluso a la adaptación externa a los cambios socio-culturales establecidos en el contexto.

\section{METODOLOGÍA}

Diseño: Se realizó una revisión organizada y sistemática de documentos de organismos científicos sobre el tema de la cultura organizacional.

Estrategia de búsqueda: En primer lugar se llevó a cabo la revisión bibliográfica y búsqueda selectiva en Google académico, Google general, Dialnet y la biblioteca de la Universidad Umecit, sobre el tema cultura organizacional de diferentes países, luego se procedió a realizar la selección de 10 trabajos de investigación: 3 tesis, 6 artículos científicos y 1 libro, dando mayor relevancia a los más recientes y pertinentes para el posterior análisis, cuya búsqueda se efectúo en español e inglés.

\section{ANÁLISIS DE RESULTADOS}

En su trabajo investigativo, Pereira, Gómez, Pinto, Ruiz y Meneses (2016) se determinó que la creatividad en las organizaciones debe implementarse a partir de la instauración de nuevas 
estrategias administrativas, de tal manera que su dinámica trascienda en el tiempo. Así mismo, la inteligencia organizacional debe responder al flujo multidireccional entre los actores, funciones, estrategias e interacciones de la experiencia compartida y el aprendizaje colectivo organizacional, ante ello, y en consecuencia, la cultura organizacional de la IES es susceptible de ser transformada, pero, en diferentes niveles (grados) y tiempos. La más próxima al cambio es la relacionada con los llamados "artefactos (lo visible y lo tangible) lo más difícil de cambiar debido al nivel de complejidad son los valores e hipótesis que exigen más inversión de tiempo, esfuerzo y compromiso para el logro de una transformación, por lo tanto, requiere emplear esquemas complejos que privilegien y estimulen elementos de liderazgo y de transformación.

Ante lo expresado, el trabajo investigativo de Pereira, et al (2016) expresa en forma clara la relevancia de esta cultura, como elemento generador de una transformación hacia el interior de las instituciones educativas, siendo más fácil transformar lo tangible u observable como las actitudes de las personas. Lo más difícil radica en la parte axiológica, es decir, los valores ético-morales, ya que estos ameritan un cambio de pensamiento en pro de ser aprendidos y aplicados en contextos determinados, para lo cual se requiere propiciar un trabajo pedagógico acorde con las necesidades o expectativas de las instituciones y la sociedad, lo que demuestra la importancia de la cultura organizacional en el campo de la educación, porque puede promover cambio de actitudes junto a la aprehensión de valores éticos y morales por parte de todos las personas involucradas.

Por otro lado, una investigación de Olivera (2015) desarrollada en la Universidad Tecnológica de Bolivar con el objetivo de diseñar un modelo de cultura organizacional para el fomento de procesos de innovación en IES, por medio de un estudio descriptivo en el que se vislumbre su función, la generación de estrategias o planes de intervención para fortalecer las competencias más débiles, con el propósito de generar espacios activos de participación y mejoramiento del aprendizaje y la socialización. 
El estudio de Olivera (2015) determinó como resultados que la Corporación Universitaria Rafael Nuñez (programas tecnológicos) debe estructurar en sus procesos académicos y administrativos un espacio para la promoción de la innovación y convertir esta actividad en un elemento fundamental del quehacer práctico, para lo cual, es necesario la creación de una cultura organizacional que fortalezca la relación entre el direccionamiento estratégico y los objetivos planteados por ésta con las actividades para gestionar la innovación.

Ante lo expuesto, el anterior trabajo de investigación se constituyó como propuesta valiosa de un modelo de cultura organizacional, que permite y promueve la innovación, con la finalidad de brindar resultados positivos en la Educación Superior. Con lo cual es posible deducir que la Cultura Organizacional es fundamental para el desarrollo de las actividades académicas en la Universidad.

Un estudio de Llanos (2018) plantea que en la actualidad las instituciones de Educación Superior se enfrentan a innumerables retos ocasionados por el mundo globalizado, estos organismos tienen como iniciativa alcanzar la calidad educativa, ante ello, se hace fundamental diagnosticar la cultura organizacional como uno de los principales promotores del desarrollo de calidad en la educación superior, ante la posibilidad de alcanzar la acreditación Institucional. Los resultados de la investigación concluyen que dicha cultura, genera identidad y da carácter a las Instituciones a nivel mundial, ya que las universidades deben cumplir con las exigencias de calidad por lo que se requiere fortalecerlas en todos sus procesos.

Del mencionado estudio se concluye que la universidad como institución de educación superior se ve abocada a desarrollar una cultura organizacional para mejorar su calidad educativa, ya que a esta le compete tener muy presente el entorno socio-cultural para desarrollar y promocionar procesos educativos acorde con las realidades de las comunidades y regiones donde esta cumple sus funciones educativas. 
Los anteriores documentos indican y hacen relación a la importancia de la cultura organizacional en las instituciones educativas, allí se hace énfasis en la relevancia de dicha cultura en el desarrollo de las organizaciones educativas, el cual es fundamental en el proceso de enseñanzaaprendizaje por la interdependencia de las instituciones educativas con el contexto social en que estas se desarrollan.

Al respecto, Cola (2020) plantea "la concepción de cultura como parte esencial en la vida organizacional, una variable influyente en el comportamiento empresarial, como también lo es el clima organizacional, los conflictos o el liderazgo" (p. 15). De lo cual es posible inferir que tal concepto es parte fundamental de la organización en cualquier empresa, incluidas las instituciones educativas, quienes deberán liderar un proceso educativo teniendo en cuenta el contexto social, puesto que su visión, misión y lineamientos curriculares se logran a partir del cumplimiento de los objetivos propuestos en el PEI llevado a cabo por parte de los profesores.

Además de los estudios indicados, González, Ochoa y Celaya (2015) plantean que en las instituciones educativas de orden superior y de bachillerato se debe precisar una cultura organizacional congruente, es decir, coherente con el diseño de estrategias compartidas, donde lo importante es diseñar políticas encaminadas al desarrollo de valores, objetivos y una misión que esté en capacidad de unificarse con la comunidad educativa, basada en identidad socio-cultural propia, por eso, la relevancia de trabajarse a partir de una cultura organizacional que promueva altos estándares de calidad.

Por otro lado, Guerrero y Silva (2017) exponen que la cultura organizacional ha adquirido gran importancia en la investigación del comportamiento de las instituciones y los resultados obtenidos por las mismas. De acuerdo a Porter (2006) dicha cultura es un elemento fundamental para la planificación estratégica de las instituciones, permite la diferenciación entre las empresas, brinda ventajas en sentido competitivo, por consiguiente, se identifica como una serie de rituales y formas 
que comparten los miembros de una organización, dando valor diferenciador, además, indica a los empleados o docentes sobre la manera de cómo se deben comportar dentro de las instituciones.

El citado estudio concluyó que las culturas desarrollan compromisos con los valores institucionales o la filosofía de la gestión lo que alentará a los empleados a trabajar por algo que creen, llevando las culturas a servir como generador de una mayor productividad, rentabilidad, e incluso por otro lado, ser sistema de control en las instituciones, gracias a que de ellas se derivan las normas y comportamientos, por ello, es importante su aplicación y promoción.

Adicionalmente, Palomino (2017) hace referencia a la importancia en la relación adelantada desde la gerencia con la cultura organizacional en pro del desarrollo institucional, como lo son las escuelas o universidades. Palomino (2017) establece que las buenas gerencias deben estar pendientes de las acciones, planes y estrategias que permitan a las empresas hacer parte de los escenarios donde se desea favorecer procesos en beneficio de la sociedad, para un buen desarrollo de las organizaciones.

Para Palomino (2017) la finalidad de una buena administración en el proceso organizacional, radica en asegurar que la energía de sus integrantes contribuya positiva y constantemente con los procesos institucionales, además, tener en cuenta las actividades prioritarias de la gerencia o administración, los esfuerzos visibles, por consiguiente, el programa institucional debe ser aplicado en forma independiente y en totalidad según el modelo que se esté siguiendo, de ahí su importancia.

\section{DISCUSIÓN Y CONCLUSIÓNES}

Las investigaciones desarrolladas por Pereira, Gómez, Pinto, Ruiz y Meneses (2016), Olivera (2015), Llanos (2018), Cola (2020) y de González, Ochoa, Celaya (2015), Guerrero y Silva (2017) y Palomino (2017) hacen entrever lo categórico de tener presente la conceptualización y desarrollo del concepto de cultura organizacional hacia el interior de las instituciones educativas, que deben ser 
consideradas como organizaciones o empresas. Además, es importante tener presente que para el desarrollo de las instituciones educativas es relevante efectuar un diagnóstico sobre el desarrollo de la misma, con base en la aplicación de técnicas cuantitativas como las encuestas o técnicas cualitativas como las sesiones de grupo, de cuya aplicación se obtendrán importantes resultados, susceptibles de análisis por medio de la comprensión e interpretación de la información alcanzada, por tanto, los resultados obtenidos son índices que permiten el diseño de las alternativas de solución para el mejoramiento de cada uno de los problemas presentados en los procesos institucionales, por consiguiente, el concientizar a los diferentes cuerpos de docentes y directivas sobre la relevancia y aplicación de una acertada cultura organizacional a nivel institucional, es definitivo, para generar un clima organizacional acorde a los requerimientos de las instituciones educativas relacionándolo con el contexto actual.

Además, es relevante que las instituciones educativas promuevan hacia el interior de las mismas la cultura organizacional, porque ella permite la creación de significados desde el punto de vista sociológico que permite la concreción de tipo social, es decir, permite como lo expuso Schein (1988) una coherencia interna de cada uno de sus miembros quienes deben incorporarse a las instituciones a través de prácticas repetitivas que hacen parte de la misma cultura creando signos y significados latentes inmersos en dicha organización, cuyos miembros la aceptan y trabajan bajo esa forma de vida haciéndose latente con el paso del tiempo y transmisible a los nuevos miembros quienes deberán acoplarse a la ideología institucional inserta en una comunidad o institución haciéndose parte constitutiva e integral de la vida de esas personas.

De igual manera, Guerrero (2017) expone que la cultura organizacional en el desarrollo de las instituciones donde están insertos los colegios y universidades cada vez adquiere más relevancia, porque se constituye en un elemento definitivo en la planificación sistemática de las organizaciones (Porter, 2006 citado en Guerrero, 2017) pus su desarrollo e implementación permiten el desarrollo de una ventaja en sentido competitivo que redundará en la calidad de la empresa u organización 
educativa, por lo tanto, autores como Robbins, Judge, Millett, y Boyle (2013 citados en Guerrero, 2017) sostienen que la cultura organizacional se la puede definiré como una serie de ritos y costumbres que comparten los integrantes de una institución u organización los cuales se convierten en el elemento que describe el ambiente en que se vive, además, de servir de guía para las nuevas personas que se incorporen a dicha institución y determinará la forma de comportamiento (Ruíz y Naranjo, 2012 citados en Guerrero, 2017).

Finalmente, es posible concluir que lo más importante para una institución educativa es tener un buen clima organizacional donde las metas estructurales de la organización deben ser conocidas por todos los integrantes o actores participes de los procesos educativos, cuyo accionar pedagógico debe encaminarse al mejoramiento de la calidad educativa, reflejada en óptimos aprendizajes y en beneficio de los estudiantes, quienes son la razón de ser en dichas instituciones.

\section{Referencias bibliográficas}

Arévalo. J. Navarro. G. y Bayona. R. (2016) La necesidad de medir la cultura organizacional.

Una revisión tel tema. Recuperado de revistas.ufps.edu.co/index.php/ingenio/article/view/2124/2064.

Barney. J.B.(1986). Organizational Culture: Can It Be a Source of Sustained Competitive Advantage? Academy of Management Review, 11 (3), 656-665. Recuperado de http://amr.aom.org/content/11/3/656. Abstract.

Cola. E. (2020). Resumen Módulo 1. Introducción al desarrollo organizacional. Panamá. UMECIT. 
De Medina. M.G. (2008). Cultura y clima en las organizaciones educativas, factor determinante en la eficacia del personal docente. Quaderns Digitals: Revista de Nuevas Tecnologías y Sociedad, (52), 14.

Esquirol. J. Sánchez. J. y Santamaría. I. (2017). La revisión bibliográfica base de la investigación. Recuperado de file:///C:/Users/ACER/Downloads/Revisionbiblbasedelainvestigacines\%20(4).pdf

Fernández. J.M. (2002). Cultura de la organización y centro educativo. Universidad Complutense de Madrid.

Gómez. E. Navas. D. Aponte. G. y Betancourth. L. (2014). Metodología para la revisión bibliográfica y la gestión de información de temas científicos a través de su estructuración y sistematización. Recuperado de file://C:/Users/ACER/Downloads/DialnetMetodologiaParaLaRevisionBibliograficaYLaGestionDe-4717293.pdf

Guerrero. M. (2017). La cultura organizacional, su importancia en el desarrollo de las empresas. Recuperado de file://C:/Users/ACER/Downloads/DialnetLaCulturaOrganizacionalSuImportanciaEnEIDesarrollo-6057495\%20(2).pdf

Gutiérrez. J. (2015). Revisión de la Literatura acerca de la cultura organizacional en el ámbito educativo. Recuperado de http://repositorio.uees.edu.ec/bitstream/123456789/2296/1/Maestrante\%20Ing.\%20Jazm\%C 3\%ADn\%20Andrade\%20Guti\%C3\%A9rrez\%20-

\%20Trabajo\%20de\%20titulaci\%C3\%B3n\%20\%20Revisi\%C3\%B3n\%20de\%20la\%20litera tura\%20acerca\%20de\%20la\%20Cultura\%20Organiza 1.pdf

Guerrero. M. y Silva. D. (2017). La Cultura Organizacional, su importancia en el desarrollo de las empresas. Recuperado de C:/Users/ACER/Downloads/DialnetLaCulturaOrganizacionalSuImportanciaEnElDesarrollo-6057495\%20(1).pdf

Izaguirre. R. Rivera. R. y Mustelier. S. (2010). La revisión bibliográfica como paso lógico y método de la Investigación Científica. Recuperado de seviciospublicos.files.wordpress.com/2010/04/revis.pdf

Llanos (2018). La cultura organizacional, principal propulsor de la calidad en Instituciones de Educación Superior: caso universidad ecuatoriana. Recuperado de revistaespacios.com/a18v39n23/a18v39n23p29.pdf 
Olivera. G. (2015). Propuesta de un modelo de cultura organizacional para la innovación en las instituciones de educación superior. Estudio de caso: programas tecnológicos de la corporación universitaria Rafael Núñez. Recuperado de https://repositorio.utb.edu.co/bitstream/handle/20.500.12585/3339/0067608.pdf?sequence= 1

Palomino. J.A. (2017). Desarrollo organizacional: teorías, prácticas y casos. Pearson. Educación.

Pereira. M. Gómez. C. Pinto. M. Ruiz. M. y Meneses. A. (2016). Transformación e innovación de la cultura organizacional en instituciones de educación superior. Estado del arte y bases conceptuales. Recuperado de file:///C:/Users/ACER/Downloads/6774Texto\%20del\%20art\%C3\%ADculo-34126-1-10-20171006.pdf

Rodríguez. R. (2009). La cultura organizacional un potencial activo estratégico desde la perspectiva de la administración. Recuperado de https://www.redalyc.org/pdf/877/87722106.pdf

Ruíz. Y. y Naranjo. J. (2012). La investigación sobre cultura organizacional en Colombia: Una mirada desde la difusión en revistas científicas. Diversitas: Perspectivas en Psicología, vol. 8, núm.2, 2012, pp. 285 - 307, 285- 307.

Sánchez. M., De la Garza. M. (2013) Cultura y estrategia en las instituciones de Educación superior.

Schein. E. H. (2004). Organizational Culture and Leadership.

Schein. E. H. (1988). La cultura empresarial y el liderazgo. Barcelona. Plaza \& Janés.

Smircich. L. (1983). Concepts of culture and organizational analysis. Administrative Science Quarterly, 339- 358. 